\title{
Una obra sobre las dinámicas familiares en México
}

\author{
García, Brígida y Orlandina de Oliveira (2006), Las familias eN \\ el MÉxico METROPOLITANO: VISIONES FEMENINAS Y MASCULINAS, 300 PP. \\ El Colegio de MéXico, MéXico, \\ ISBN: 968-121-229-0
}

\begin{abstract}
El hecho de privilegiar el hogar como unidad de análisis permite ubicar la cuestión de las identidades, como una condición vivida por una persona y por los grupos en el ámbito donde se producen y reproducen las relaciones sociales de naturaleza intima: las familias.

Vania Salles (1992).
\end{abstract}

\section{Introducción}

La investigación sobre la dinámica intrafamiliar como un espacio cotidiano de la reproducción generacional muestra sistemas de acción persistentes en tanto que los hábitos y trabajos del ámbito doméstico se fundamentan en una representación y valoración social, según género, tradición familiar y sector social, que los articula a estructuras relativamente consolidadas. El trabajo de García y Oliveira se pregunta, entre muchos interrogantes y desde un enfoque de género, sobre las permanencias y modificaciones masculinas y femeninas que ocurren en la convivencia y los arreglos de la vida familiar.

El libro presenta una revisión exhaustiva de las hipótesis sobre la dinámica intrafamiliar en México -con validez para el contexto latinoamericano-, y su confrontación con información empírica original, lo que les permitió a las autoras mostrar los arreglos actuales de la familia metropolitana -México y Monterrey- de los sectores medios y populares urbanos.

Analizan la realidad intrafamiliar desde la información recogida en la Dinaf, ${ }^{1}$ la cual consta de un cuestionario de hogar y dos muestras individuales, y la presentan en cuatro capítulos centrales,

\footnotetext{
${ }^{1}$ Encuesta sobre dinámica familiar diseñada por las autoras y aplicada a una muestra de hogares por el Instituto Nacional de Estadística, Geografía e Informática (INEGI) entre fines de 1998 y principios de 1999.
} 
desarrollando cada uno de ellos en dos partes: las contribuciones hechas a las diferentes dimensiones incluidas, y el análisis de los datos construidos, de tal manera que muestran los cambios de esas dinámicas familiares en proceso. Teniendo en cuenta los apartados centrales, el libro se estructura en siete capítulos. El primero es una revisión analítica de las teorías sobre la familia y de los roles de género inscritos en dichas visiones, mientras que en el segundo nos ubican en los contextos metropolitanos de México, Distrito Federal, y Monterrey en un doble esfuerzo: destacando las características sociodemográficas y económicas dominantes de la población y la región en cada una de esas ciudades, y comparando estas últimas en su articulación con la estructura de los mercados de trabajo.

Puedo entrever que las preguntas que guiaron el libro tienen una doble fuente: histórica, desde la investigación de las autoras sobre la dinámica familiar en México que empieza a hacerse visible a inicios de la década de 1980 (Muñoz et al., 1978, 1979, y 1981; García et al., 1982; Oliveira y Salles, 1989), y los cuestionamientos mas recientes sobre los cambios parciales y progresivos que ellas mismas han registrado en determinados cortes temporales (García y Oliveiras, 2004a y 2004b; Oliveira, 1995 y 1998; Oliveira y García, 1990a, 1990b, 1992 y 1994; Oliveira et al., 1989; Oliveira y Salles, 1989). Las preguntas vigilan una crítica a la literatura especializada y los propios resultados anteriores, argumentando el diseño inacabado de la familia y proponiendo una relectura desde los nuevos arreglos de género en la convivencia conyugal, doméstica y de paternidad que finalmente fraguan la vida familiar.

De su trayectoria de investigación en esta línea, García y Oliveira mantienen las preocupaciones sobre el impacto de la estructura de los mercados de trabajo en la reproducción familiar según sectores sociales; el impacto de los periodos de crisis económica en la mayor exposición de las mujeres al trabajo extradoméstico remunerado; el vínculo entre escolaridad, tipo de trabajo y salario; el vínculo entre composición sociodemográfica de los cónyuges, tamaño de familia, edad de la unión y evolución del ciclo vital familiar. Han analizado la relación entre estos indicadores a la luz de las modalidades que va tomando la organización familiar como: el modelo patriarcal de familia, la jefatura femenina, la unidad nuclear, la familia extensa, la familia monoparental, las unipersonales, las unidades compuestas, la organización corresidencial y la no corresidencial, en el supuesto sociodemográfico y sociológico de la sobrevivencia familiar y económica. 
En su preocupación más actual, incorporan la importancia discriminada por género de la participación económica en la reproducción cotidiana, las percepciones masculinas y femeninas en el supuesto proyecto unificado de familia, los factores intervinientes en las dinámicas de violencia conyugal y familiar que derivan de la tradición de la familia de origen en cada uno de los cónyuges, y de las formas de resolver la relación y los comportamientos intergénero en la familia de procreación; asimismo, los factores que favorecen la autonomía de la mujer en la relación de pareja y de familia, tanto como los que la retrasan. Dar prioridad al análisis de la relación de pareja y familia en el escenario de la relación social intergénero, y observar la relación familiar en su doble contenido, como relación social y de parentesco intergénero e intergeneraciones, les permitió, desde hace varios años, revisar contenidos preestablecidos de esas relaciones e incorporar progresivamente los 'matices' que invalidan las rigideces que explicaban el funcionamiento de los modelos familiares dominantes -de ahí que fuera posible que esos matices quedaran propuestos para ser recabados en la cédula de la Dinaf de 1998-1999.

Vinculado con lo anterior, García y Oliveira incorporan en esta investigación la dinámica estructural más reciente de los mercados de trabajo en cuanto que éstos se inscriben en el horizonte de la reestructuración y flexibilidad laboral, lo que impacta significativamente en la reformulación de las estrategias de organización social.

En consecuencia, las autoras nos hacen una advertencia: la contemporaneidad de los procesos sociales se registra también en una multirrelación segmentada de los determinantes casuísticos que perfilan conexiones específicas en las distintas configuraciones de la realidad, por lo que no se puede ocultar el movimiento de la pluralidad ni soslayar el complejo desplazamiento de algunos elementos definitorios de un sistema de familia a otros diseños, que a su vez cobran insistentemente relevancias que no se agotan en una intimidad relativamente conocida.

Así, el análisis de estas relaciones se expone como resultados y hallazgos en los siguientes capítulos del libro: III. Dinámica intrafamiliar en hogares con jefatura masculina; IV. Mujeres jefas de hogar y su dinámica intrafamiliar; v. El ejercicio de la paternidad; y vi. El trabajo extradoméstico femenino y las relaciones de género en la pareja.

En el último capítulo, aparte de resaltarse las conclusiones, se sugiere una reflexión sobre la necesidad de supervisar la vigen- 
cia de las explicaciones en la dinámica intrafamiliar con imperativos en sus modelos de funcionamiento, como "los múltiples factores contextuales, individuales y familiares" que otorgan desde las movilizaciones socioculturales el tono de las transformaciones familiares; por ejemplo: el incremento de hogares con múltiples proveedores con su correlato de jefatura compartida y sus repercusiones en el anterior modelo del varón-único proveedor, por señalar sólo un rasgo sistémico que se deblita o desaparece en una variante de arreglo familiar.

En esta reseña sólo puedo señalar y resaltar algunos de los hallazgos que las autoras encontraron en la familia metropolitana y que vivencian los protagonistas como parte de las 'persistencias y reacomodos' en el proceso de organización social-familiar actual; hallazgos que, a su vez, nos obligan nuevas preguntas y reflexiones. El señalamiento de los hallazgos no debe verse como un nivel descriptivo del libro, sino como el intento de señalar el esfuerzo multirrelacional que en cada uno de los capítulos permite mostrar los diferentes ejes de la dinámica familiar, lo que hace significativamente novedoso este libro en la investigación sobre dinámica intrafamiliar en México.

\section{El método}

En virtud de que la investigación está dirigida a medir la dinámica intrafamiliar, se acota, entre las categorías centrales, la 'unidad familiar' con: capacidad de organización doméstica dirigida a garantizar la reproducción cotidiana y generacional de sus integrantes; con una estructura jerárquica y de poder que establece formas de convivencia develando roles de parentesco, género y generación, y con un conjunto axiológico compartido en función de elecciones y acciones cotidianas.

Las ‘percepciones', están vinculadas con el 'género' en cuanto a las formas actuar en la relación de pareja, en las relaciones entre padres e hijos, en la mayoría de los eventos de la cotidianidad familiar: la dinámica afectiva y sexual de los cónyuges, los trabajos domésticos, el cuidado de los hijos. En la encuesta se buscó captar, entre otras variables, información sobre la participación masculina en la vida doméstica y sobre, las percepciones desde la perspectiva de ambos cónyuges, de tal modo que quedaron consignadas diferencialmente en cada una de las variantes de organización familiar analizadas: con jefatura masculina o femenina. 
Como ya mencionamos, la investigación se desarrolló en dos áreas metropolitanas: México y Monterrey. Incluyó varios recursos de información original y de análisis de la misma.

Básicamente, la información empírica se obtuvo de la Encuesta sobre dinámica familiar Dinaf, aplicada a hogares, y de dos muestras individuales dirigidas a hombres $(1,644)$ y mujeres $(2,532)$ de 20 a 50 años. Las variables centrales se agruparon en variables sociodemográficas, socioeconómicas y percepciones, lo cual permitió cubrir un horizonte de información 'objetiva y subjetiva' en la encuesta Dinaf y en las muestras individuales. Observo que una parte importante de las variables involucradas en la muestra general y en las muestras individuales ha sido probada en otras investigaciones de y por las autoras, lo que les permitió hacer referencia al comportamiento de las dinámicas intrafamiliares en México en un periodo de por lo menos 25 años de análisis con investigación e información primaria y encuestas probabilísticas. Todo lo anterior indica que el fundamento teórico-metodológico de este libro está tejido también en los antecedentes de su propia obra.

\section{Diversos recursos de análisis estadísticos}

Se utilizaron varios recursos estadísticos para el análisis de la información. Las diferentes unidades de análisis que arrojaron los distintos arreglos familiares -los hogares con jefatura masculina, con jefatura femenina, el ejercicio de la paternidad, el trabajo extradoméstico femenino- fueron construidas estadísticamente por medio de índices sumatorios y posteriormente análisis de clasificación múltiple -lo que permitió controlar la distinta composición sociodemográfica de las dos muestras individuales y observar las variaciones dadas por el género, en cuanto a las percepciones de los jefes y de las esposas, y de las jefas de hogar. En algunos casos se utilizó análisis multivariado, regresión logísitica y variables dicotómicas -en el caso del trabajo extradoméstico femenino, por ejemplo-, y se construyeron modelos para explicar la proporción de variación en las diferentes dimensiones analizadas.

Las variables de control se agruparon en tres grupos de factores: "los relativos a los/las entrevistados (edad, diferencia etárea entre los cónyuges, participación laboral de la mujer, sector social y lugar de residencia), los relativos a las familias actuales (otro adulto en el hogar, diferente a los cónyuges), y los relativos 
a las familias de origen (condición económica y residencia en la niñez)". Las características sociodemográficas de las esposas y de sus familias de procreación fueron fundamentales como variables de control en los modelos estadísticos.

\section{Los hallazgos}

El análisis de la dinámica intrafamiliar 'con jefatura masculina', corresidencial, se hizo a partir de resultados de muestras independientes de varones-jefes y mujeres-esposas, no parejas entre sí, centrándose el análisis en las percepciones masculinas y femeninas en cuanto a: la forma de convivencia en la 'relación de pareja' y 'relaciones entre padres e hijos', la división de los trabajos reproductivos, y las concepciones respecto a los roles de género.

Los hallazgos corroboraron una participación masculina diferencial según el tipo de trabajo doméstico. Las percepciones directas indicaron algunas coincidencias y discrepancias entre las versiones de ambos cónyuges. Los varones creen tener una mayor participación en los trabajos reproductivos y en las decisiones familiares de lo que declaran las mujeres sobre sus cónyuges. Paralelo a ello, los hombres subestiman la participación de las esposas frente a la autocalificación de ellas en los mismos ámbitos. La toma de decisiones aún está vinculada con roles de género preestablecidos como 'adecuados' para hombres y mujeres.

La pertenencia a un sector de clase es una mediación significativa. Se resaltan que los sectores medios han sido más permeables a nuevas formas de organización y convivencia familiar, distanciándose de modelos tradicionales que exhiben distintos grados de subordinación femenina. Lo contrario se encuentra en los sectores populares, en los que persiste mayor rigidez en relaciones autoritarias por parte del jefe varón, que mantiene una relación de iniquidad y subordinación hacia el resto de los miembros, particularmente de la mujer cónyuge. En este hallazgo también interviene la variable edad, que muestra que en el caso de los sectores populares, la relación autoritaria tiende a disminuir con el avance de la edad.

La hipótesis de la persistencia en el mantenimiento de pautas tradicionales, y una valoración jerárquica asociada con el género, de los oficios 'necesarios', definidos como tales en la construcción social, fue confirmada por ejemplo, en una restringida participación masculina en algunas actividades reproductivas o 'prestación de servicios domésticos, y en un relativo mayor apoyo en los trámites externos o 'servicios de apoyo', las que represen- 
tan acciones mas neutrales que el patrón de 'oficios' acuñado a la 'madre' y a la 'mujer'. Paralelamente, se observó una tendencia al cambio en las relaciones con los hijos proveniente de mayores tiempos y actividades compartidas en el ejercicio de la paternidad, lo que podría verse como uno de los indicadores de "nuevas formas de organización y convivencia familiar estructuradas en relaciones menos asimétricas".

Se confirma que los roles masculinos ligados a la figura del 'hombre y único proveedor' se ven relativizados por la inserción laboral de la mujer y de otros miembros del hogar, y, en consecuencia, la 'autoridad paterna' de corte patriarcal se ve matizada, por relaciones de convivencia un tanto democráticas.

En este capítulo, particularmente se inicia un examen sobre uno de los ejes importantes y poco analizados de la dinámica de un tipo de familia: las percepciones sobre la autoridad del hombre y/o padre en proceso de cambio ante las transformaciones en la organización interna de la familia. Aunque se trata la relativización de la autoridad del padre desde: la capacidad exclusiva (o no) de la toma de algunas decisiones familiares, su incidencia en la relación de pareja en cuanto al 'permiso' o los 'permisos', y la violencia de pareja, se deja abierto el análisis sobre la percepción social de la masculinidad y la jefatura masculina. En la construcción social de género, ¿en el hombre todavía se prefigura el rol potencial de ser jefe del hogar? En función de los ámbitos de frustración masculina ante la autoridad compartida, autoridad segmentada, o jefatura femenina en pareja corresidencial, ¿̇uáles son los espacios de reformulación de la representación masculina en la familia, según los sectores de clase considerados?

Por otro lado, quedan también por ser explorados los conflictos de pareja generados por las uniones de facto paralelas a la pareja familiar y su impacto sobre la jefatura masculina.

El análisis de la dinámica familiar 'con jefatura femenina' corrobora algunos hallazgos que se han confirmado en América Latina y en México en particular. El incremento de la 'jefatura femenina' en la región nos ubica ante una variante familiar en transformación (García y Rojas, 2002). Entre 1990 y 2000, México tiene $21 \%$ de los hogares en esta jefatura. Algunos aspectos sociodemográficos y socioeconómicos característicos de estos hogares son: el menor tamaño y número de integrantes, unidades extendidas, mayor número de perceptores económicos que contribuyen el gasto final de la reproducción cotidiana (García y Rojas, 2002). Por otra parte, también está cada vez más estudia- 
da la relación entre jefatura femenina y pobreza (Rubalcava y Murillo, 2001, 2002 y 2005; Salles, 2000).

La jefatura femenina se registra a partir del reconocimiento de los integrantes de la unidad doméstica como, jefatura declara$d a$, lo cual superó un problema metodológico para la investigación sociodemográfica y ha devenido en un incremento de un reconocimiento social que contribuye a superar el subregistro encubierto por la nominación patriarcal. Éste es un aporte y una decisión anterior a esta publicación.

A partir de la Dinaf, se encontró que 14\% de las mujeres eran 'jefas de hogar'. Dicha condición deviene de relaciones conyugales "que concluyen en divorcio, separaciones, viudez, o arreglos de pareja no corresidenciales”. El análisis de los arreglos domésticos en familias con ese tipo de jefatura fueron hechos con base en la información directa de las mujeres y de otros miembros del hogar, lo que fortalece la dimensión de percepciones por género más allá de los cónyuges varones.

Algunas características halladas y que a su vez confirman el perfil familiar de las 'jefas' en México, fueron: mujeres económicamente activas y con trayectoria laboral; su salario no es el único ingreso que solventa el gasto familiar, pues a éste se le suma, por lo menos, una parte de los ingresos de los otros perceptores y otros apoyos eventuales; los trabajos más recurrentes se registraron en servicios personales y como asalariadas. El relativo nivel de bienestar se obtiene por la multiplicación de ingresos, a los que se recurre como parte de las estrategias familiares de sobrevivencia, pues este grupo de mujeres y sus familias se registraron más en el universo de los sectores populares y pobres, aunque no sean ellas las más pobres.

Incluir a otros miembros de la unidad doméstica en la medición y registro de los arreglos intrafamiliares debe considerarse como una contribución a la investigación de la realidad familiar, pues los antecedentes son escasos y parciales. En cuanto a los arreglos de las prácticas reproductivas en este tipo de hogares, se confirma la mayor responsabilidad y decisión en cabeza de la mujer jefa, mostrándose que no hay una participación más activa e igualitaria de los otros miembros, y que se mantiene la sobrecarga de la jornada femenina con los trabajos más pesados que requieren mayor tiempo reproductivo. Lo anterior indica que sigue manteniéndose un rígida asociación y valoración de las tareas domésticas por género, vinculadas con las supuestas responsabilidades de parentesco, por ejemplo, en la figura de la 
madre y/o jefa. Quedan sin explorar ámbitos de la dinámica intrafamiliar que quizá inviten una participación más equitativa intergénero e intergeneraciones; por ejemplo, todo lo referente a las relaciones sociales familiares -festejos, visitas o frecuencias de allegados cotidianos a la vida- familiar y otros escenarios de socialización cotidiana.

Las autoras señalan que las 'jefas' tienen al interior de su unidad doméstica, mayor capacidad de decisión y 'la última palabra' en una buena parte de las decisiones que refieren a la jornada reproductiva y a su propio trabajo extradoméstico. Sería interesante, a partir de las percepciones, continuar captando el sentir de responsabilidad que significan las decisiones familiares como parte de la carga emocional para la jefa, puesto que por, ejemplo, impulsar a un miembro joven del hogar a la dinámica laboral puede significar un cambio de perspectivas sobre la formación de la fuerza de trabajo individual y familiar.

La relación entre jefatura femenina y violencia doméstica es analizada a partir de información que captó episodios relativos a la infancia en la familia de origen, y a la vida adulta en la familia de procreación, incluyendo la relación de pareja y la relación de paternidad. Ante la ausencia de la pareja que suponen el divorcio, la separación o la viudez, lo que queda pendiente es: ¿a qué tipo de pareja se refiere la relación de violencia actual? Pareciera que a la última de convivencia permanente, pero al no quedar completamente explícito podría referirse también a parejas de facto o parejas eventuales que registren para ello alguna permanencia o comportamiento de conyugabilidad no corresidencial.

Una conclusión que confirma una relación importante es que la violencia doméstica puede mantenerse como un factor asociado con la ruptura conyugal y a la composición de hogares con jefatura femenina, y a la inversa, que la jefatura de la mujer puede ocasionar dinámicas violentas para ella al interior de la pareja, lo que sugiere inicialmente que comportamientos de autonomía de la mujer revierten negativamente en su dinámica social, afectiva y sexual al interior de la familia y pareja.

Otras variables que se incluyeron en el impacto de mayor riesgo de la mujer a estar expuesta a dinámicas de violencia y maltrato intrafamiliar por parte del cónyuge son: el sector social de pertenencia, y la presencia de otra mujer adulta. De hecho, lo que se confirma es que las mujeres jefas referidas pertenecen en mayor medida a sectores populares y con experiencia de maltrato en la familia de origen y en la familia actual. El contexto de escasez eco- 
nómica, niveles educativos básicos o inexistentes, vinculación laboral inestable, organización tradicional de autoridad y tiranía paterna, y otras situaciones asociadas, puede favorecer y fortalecer un sistema de agresión contra las mujeres cónyuges e hijas que puede mantenerse en la vida adulta y de pareja como un elemento que predispone un hábito emocional de permitir o soportar el maltrato.

Estos resultados de las 'mujeres jefas' son presentados en contraste con los de las esposas que tienen arreglos familiares con jefatura masculina, y también son particularizados en dos contextos metropolitanos -México y Monterrey-que le otorgan un matiz diferenciador a las mismas características.

El análisis del 'ejercicio de la paternidad' es un aporte en proceso cuyo objetivo central fue documentar la participación del varón en la vida familiar y reproductiva, más allá de la condición tradicional de éste como proveedor económico y representante de la autoridad familiar. Indagar sobre el cuidado, cercanía y afecto entre padres e hijos/as, requirió vincular de nuevo 'condiciones individuales, familiares y contextuales' de los hombres encuestados -Dinaf muestra individual a varones-, y, sobre todo, tocar un aspecto que tradicionalmente había sido adjudicado al rol de la mujer. Es decir que se midió el posible cambio en un rol de género, uno de los ejes analíticos centrales de la investigación. Uno de los hallazgos fue que sólo la tercera parte de los entrevistados declaró una atención más directa a los hijos e hijas consistente en el cuidado a los mismos y en atender el transporte básicamente escolar. El tipo de trabajo asalariado, el nivel de ingresos, la residencia en la infancia del padre, y la valoración sobre una distribución familiar más simétrica fueron antecedentes explicativos de la participación paterna en el caso de los hombres; otros elementos que favorecen esta participación en las características de la esposa son el nivel de escolaridad de ella, y su inserción en el trabajo extradoméstico, lo que en conjunto redunda en una redefinición de la división de trabajo familiar.

Debe considerarse un aporte significativo la separación analítica entre 'jefatura masculina', 'ejercicio de la paternidad' y 'percepciones masculinas’ al interior de las dinámicas familiares cotidianas, porque se logra deslindar un vínculo tradicional que tendía a preestablecer roles de género y parentesco en el análisis de la familia. ${ }^{2}$

\footnotetext{
${ }^{2}$ Algunos antecedentes de esta separación analítica han sido explorados por Pilar Gonzalbo (2006); Lévi-Straus (1995); Paul Viene (1990), David Cooper (1983), entre otros.
} 
El análisis del 'trabajo extradoméstico femenino y su impacto en las relaciones de género en la pareja' y al interior de la familia, muestra un vínculo 'significativo' que repercute en la menor subordinación de la mujer y según se confirman sus hallazgos, se deriva principalmente de: el control de los recursos económicos, la proporción del aporte de su salario al gasto familiar, la propia valoración sobre su trabajo; y el sentido de su trabajo en el proyecto personal.

En el contexto de ese vínculo, las relaciones de género fueron analizadas a partir de las siguientes dimensiones:

- La "participación los hombres en la vida familiar" según algunos tipos de tareas referentes al ámbito doméstico y a la paternidad;

- El "empoderamiento de las mujeres" a partir del trabajo extradoméstico, toma de decisiones, autonomía en el tiempo personal, y, autonomía en el gasto de sus ingresos;

- "Tipos de violencia intrafamiliar" o no violencia.

Encontraron y confirmaron que la experiencia laboral femenina es altamente significativa para explicar la mayor simetría en las relaciones de pareja, -mayor simetría que asocia un apoyo de los hombres al trabajo doméstico y al cuidado de los hijos, mayor participación de las mujeres en la toma de decisiones, gastos y libertad de movimiento, y mayor capacidad de negociación. El análisis detallado les permitió observar las variaciones de significación en las dinámicas intragénero e intrafamiliares, según la antigüedad en el trabajo, la participación del salario de ella en el aporte y gasto familiar total, el tipo de trabajo según actividad -asalariado o por cuenta propia-, la jornada del mismo, el nivel escolar, y la valoración que las mujeres otorgan a su trabajo. Esta valoración redunda en desarrollo personal, autonomía, progreso y compromiso laboral como proyecto de vida, y tiene una incidencia importante en la explicación de los niveles de negociación en los arreglos intrafamiliares e intergénero menos desiguales.

El empoderamiento de las mujeres se ve matizado con la relación de subordinación que indica el 'permiso' de los hombres para que las mujeres realicen actividades cotidianas -externas a la familia, y algunas relacionadas con la vida familiar pero externas a la vivienda-. Así, se encontró que la relación intergénero alrededor del permiso se mantiene en aproximadamente dos tercios de las mujeres que no trabajan, y en un poco más de la mitad 
en las mujeres insertas laboralmente. La solicitud del permiso disminuye en relación con la mayor importancia de 'autonomía y desarrollo personal e individual' que la mujer le da a su trabajo extradoméstico. En momentos anteriores, las autoras han explorado la figura del 'permiso' como un rasgo de autoridad patriarcal y como un rasgo cultural que se conjuga en la construcción de género y de relaciones intergénero. Ubicarlo ahora como parte de la negociación y de los logros hacia el empoderamiento de la mujer en la dinámica intrafamiliar, e intersectores sociales, es confirmar su ubicación en el tejido de las relaciones sociales y, en consecuencia, lograr un desplazamiento analítico disciplinario hacia la sociología.

En cuanto a la dinámica de violencia intrafamiliar, los hallazgos confirmaron que las mujeres vivenciaron una mayor incidencia de ésta en la familia de origen que en la familia de procreación. Es decir, los rasgos sociodemográficos antecedentes de las esposas en la familia de origen, tanto como en las de procreación, son factores explicativos de las relaciones de género en la pareja, sobre todo referidos a las dinámicas y tipos de violencia que ella aceptan.

Como el lector podrá intuir, estamos frente a la densidad de un análisis multirrelacional que, como lo advertía Weber es la condición fundamental necesaria para llegar a comprender y ordenar racionalmente la realidad empírica con el fin de establecer los procesos histórico-sociales que caracterizan y/o configuran el conjunto finito de las regularidades que emanan de las prácticas de los colectivos, en este caso, de la dinámica cotidiana de la vida y arreglos familiares en el México metropolitano.

\section{Bibliografía}

Cooper, David (1983), La muerte de la familia, Ariel, Barcelona.

García Brígida y Olga Rojas (2002), “Los hogares latinoamericanos durante la segunda mitad del siglo xx: una perspectiva sociodemográfica”, Estudios Demográficos y Urbanos, El Colegio de México, México, mayo-agosto, vol. 17 (2): 261-288.

García Brígida y Orlandina de Oliveira (2004a), "El ejercicio de la paternidad en el México urbano”, en Marina Ariza y Orlandina de Oliveira (coords.), Imágenes de la familia 
en el cambio de siglo, Instituto de Investigaciones Sociales, UNAM, pp. 283-317.

García Brígida y Orlandina de Oliveira (2004b), “Trabajo extradoméstico femenino y relaciones de género: una nueva mirada”, Estudios Demográficos y Urbanos, El Colegio de México, México, enero-abril, vol. 19 (55): 145-180.

García, Brígida; Humberto Muñoz y Orlandina de Oliveira (1982), Hogares y trabajadores en la ciudad de México, Investigaciones Sociales, Universidad Nacional Autónoma de México-El Colegio de México, México.

Gonzalbo, Pilar (2006), Introducción a la historia de la vida cotidiana, El Colegio de México, México.

Lévi-Straus, Claude (1995), Polémica sobre el origen y la universalidad de la familia, Anagrama, Barcelona.

Muñoz, Humberto; Brígida García y Orlandina de Oliveira (1979), Migración, familia y fuerza de trabajo, Cuadernos del ces, núm. 26, El Colegio de México, México.

Muñoz, Humberto; Orlandina de Oliveira y Claudio Stern (1978), Migraciones internas a la ciudad de México y su impacto sobre el mercado de trabajo, Temas de la ciudad, núm. 8, Delegación Venustiano Carranza, México.

Muñoz, Humberto; Orlandina de Oliveira y Claudio Stern (1981), Migración y desigualdad social en la ciudad de México, $2^{\mathrm{a}}$ ed., Instituto de Investigaciones Sociales, Universidad Nacional Autónoma de México-El Colegio de México, México.

Oliveira, Orlandina (1995), "Experiencias matrimoniales en México: la importancia de la familia de origen”, Estudios Sociológicos, El Colegio de México, México, mayo-agosto, XIII (38): 283-308.

Oliveira, Orlandina (1998), "Experiencias matrimoniales en México: la importancia de la familia de origen", en Vania Salles y José Manuel Valenzuela Arce (coords.), Vida familiar y 
cultura contemporánea, Col. Pensar la Cultura, ConaculTA/Dirección General de Culturas Populares e Indígenas, México.

Oliveira, Orlandina (1998), "La participación femenina en los mercados de trabajo", Trabajo, 1 (1): 139-161.

Oliveira, Orlandina y Brígida García (1990a), “Expansión del trabajo femenino y transformación social en México: 19501987", en México en el umbral del milenio, El Colegio de México, México, pp. 345-375.

Oliveira, Orlandina y Brígida García (1990b), “Trabajo, fecundidad y condición femenina en México", Estudios Demográficos y Urbanos, El Colegio de México, México, septiembre-diciembre, vol. 5 (3): 693-710.

Oliveira, Orlandina y Brígida García (1992), "Jefas de hogar y violencia doméstica”, Revista Interamericana de Sociología, mayo-diciembre, vol. 6 (2-3): 179-200.

Oliveira, Orlandina y Brígida García (1994), Trabajo femenino y vida familiar en México, El Colegio de México, México.

Oliveira, Orlandina, Bruce Christenson y Brígida García (1989), "Los múltiples condicionantes del trabajo femenino en México", Estudios Sociológicos, El Colegio de México, México, mayo-agosto, vol. 7 (20): 251-280.

Oliveira, Orlandina y Vania Salles (1989), "Introducción: Acerca del estudio de los grupos domésticos: un enfoque sociodemográfico", en Orlandina de Oliveira; Marielle Pepin Lehalleur y Vania Salles (comps.), Grupos domésticos y reproducción cotidiana, Universidad Nacional Autónoma de México-El Colegio de México-Miguel Ángel Porrúa, México, pp. 11-36.

Rubalcava, Rosa María y Sandra Murillo (2005), “El ingreso de los hogares rurales pobres y los beneficios monetarios del programa Oportunidades vistos con un perspectiva socioespacial de género: la jefatura femenina en Guanajuato", en María de la Paz López y Vania Salles (coords.), El Pro- 
grama Oportunidades examinado desde el género, Desarrrollo de las Naciones Unidas para la Mujer (UNIFEM)-El Colegio de México, México.

Rubalcava, Rosa María y Sandra Murillo (2002), “Localidades y hogares en un mundo de propensiones", Revista Argumentos, UAM-Xochimilco, agosto, 42.

Rubalcava, Rosa María y Sandra Murillo (2001), “Marginación, hogares y cohesión social”, en Mauricio de María y Campos y Georgina Sánchez (eds.), ¿Estamos unidos mexicanos? Los límites de la cohesión social en México. Informe de la sección mexicana del Club de Roma, Planeta, México.

Rubalcava, Rosa María y Sandra Murillo (1998), Necesidades, recursos y posibilidades: el ingreso de los hogares mexicanos en el periodo 1984-1994, tesis de doctorado en ciencias sociales, CIESAs-Universidad de Guadalajara, Guadalajara, México.

Salles, Vania (2000), “¿Cargan las mujeres el peso de la pobreza?”, en Vania Salles y Rodolfo Tuirán, Familia, género y pobreza, Miguel Ángel Porrúa, México, pp. 47-94.

Salles, Vania (1998), "Las familias, las culturas y las identidades”, en Vania Salles y José Manuel Valenzuela Arce (coords.), Vida familiar y cultura contemporánea, Col. Pensar la Cultura, Conaculta/Dirección General de Culturas Populares e Indígenas, México.

Salles, Vania (1992), "Las familias, las culturas y las identidades”, en José Manuel Valenzuela (comp.), Decadencia y auge de la identidades, El Colegio de la Frontera Norte, Tijuana.

Veyne, Paul (1990), "Desde el vientre materno hasta el testamento. El matrimonio, los esclavos, la familia y sus libertos”, en Philippe Ariès y Georges Duby, Historia de la vida privada, t. I, Taurus Ediciones, Madrid, pp. 23-101.

Weber, Max (1971), Sobre la teoría de las ciencias sociales, Península, Barcelona. 
Recibido: 13 de septiembre de 2006. Aceptado: 27 de octubre de4 2006.

Luz María Salazar-Cruz. Es doctora en sociología por El Colegio de México Seminario. Sus temas de investigación son: estrategias de sobrevivencia en mujeres y trayectorias laborales en mujeres. 